\title{
Synthesis, growth and characterization of organic crystal triphenylmethane p-nitroaniline: a nonlinear optical crystal
}

\author{
G. Bhuvaneswari ${ }^{1,3}$, L. Guru PrasaD ${ }^{2}$, N. PrabhaVAThi ${ }^{3, *}$ \\ ${ }^{1}$ Department of Physics, Muthayammal College of Arts and Science, Rasipuram, India \\ ${ }^{2}$ Department of Science \& Humanities, M. Kumaraswamy College of Engineering, Karur, India \\ ${ }^{3}$ Department of Physics, Sri Sarada College for Women, Salem - 636 016, India
}

\begin{abstract}
A triphenylmethane-4-nitroaniline salt has been synthesized and the crystals have been grown by using slow evaporation solution growth technique. The grown single crystal was investigated by recording the powder XRD, FT-IR spectrum, UV-Vis spectrum, Vickers microhardness test, dielectric measurements and powder SHG. The growth of crystal was confirmed by analyzing the XRD pattern and it was stated that the grown material crystallized in triclinic system. Vibrational peaks of the functional groups existing in the compound were identified. Vickers microhardness studies were carried to measure the mechanical strength. In order to identify the phase transition temperature, the dielectric studies were carried out at different temperatures. Dielectric constant, dielectric loss and impedance of the crystal were monitored with respect to the frequency of electric field.
\end{abstract}

Keywords: crystal growth; UV-Vis spectroscopy; FT-IR; XRD; dielectric studies; nonlinear optical material

\section{Introduction}

In recent days, considerable advances have been developed in the field of nonlinear optics (NLO). The development of fiber optic communication systems has stimulated the search for new high-efficiency NLO materials which are capable of processing the optical signals in fast manner. Nowadays, the NLO is getting more and more researchers' interest due to its potential applications in the field of optoelectronics and telecommunication systems [1, 2]. Many organic crystals have been reported with high nonlinear optical character because most of the organic systems are made of delocalized $\pi$ electrons which create a friendly environment to convert the frequency of electromagnetic radiation in an efficient manner [3]. Organic nonlinear materials have large optical susceptibilities, and high optical thresholds compared with their counterparts [4-7]. 4-nitroaniline (4NA) and its derivatives are among the most extensively examined NLO materials and its molecular

*E-mail: n.prabavathi@yahoo.co.in structure has donor- $\pi$-acceptor system which enables it to achieve relatively high second harmonic conversion (SHG) efficiency [8].

Triphenyl methane (TPM) is stabilized by extensive delocalization over three phenyl rings. Nevertheless, due to steric effects, delocalization of charge is not occurring at the same time on the three rings. Instead, each ring influences the other two, out of coplanarity, forming a three blade fan structure, so that the delocalization takes place in the phenyl ring if p-orbital of the anionic carbon is parallel to the p-orbital of any other phenyl ring [9] Generally, the charge transfer molecule shows high optical nonlinearity. Hence, we planned to synthesize triphenylmethane-4-nitroaniline (TPMNA) salt and to grow the crystals. In this manuscript, we gave the information about the growth and characterization of TPMNA crystals.

\section{Materials and methods}

The title material was synthesized from triphenyl methane (Alfa Aesar) and p-nitroaniline (Alfa Aesar) which were mixed 
at the stoichiometric ratio of 1:1. Triphenyl methane was dissolved in $50 \mathrm{~mL}$ of toluene and 4-nitroaniline was dissolved in acetone $(50 \mathrm{~mL})$. The dissolved triphenyl methane and nitroaniline solutions were stirred continuously using a magnetic stirrer for 2 hours. The prepared homogeneous mixture was kept undisturbed to evaporate at room temperature. Disappearance of the solvent yielded tiny crystals and this tiny crystals were purified by continual recrystallization process. The yellow crystal was harvested after 10 days. Fig. 1 shows the picture of an as-grown TPMNA crystal. The reaction involved in synthesis of TPMNA is illustrated by the following equation:

$$
\begin{aligned}
& \left(\mathrm{C}_{9} \mathrm{H}_{5}\right)_{3} \mathrm{CH}+\mathrm{O}_{2} \mathrm{NC}_{6} \mathrm{H}_{4} \mathrm{NH}_{2} \longrightarrow \\
& \left(\mathrm{C}_{9} \mathrm{H}_{5}\right)_{3} \mathrm{CH} \cdot \mathrm{C}_{6} \mathrm{H}_{4} \mathrm{NH}_{2} \mathrm{NO}_{2}
\end{aligned}
$$

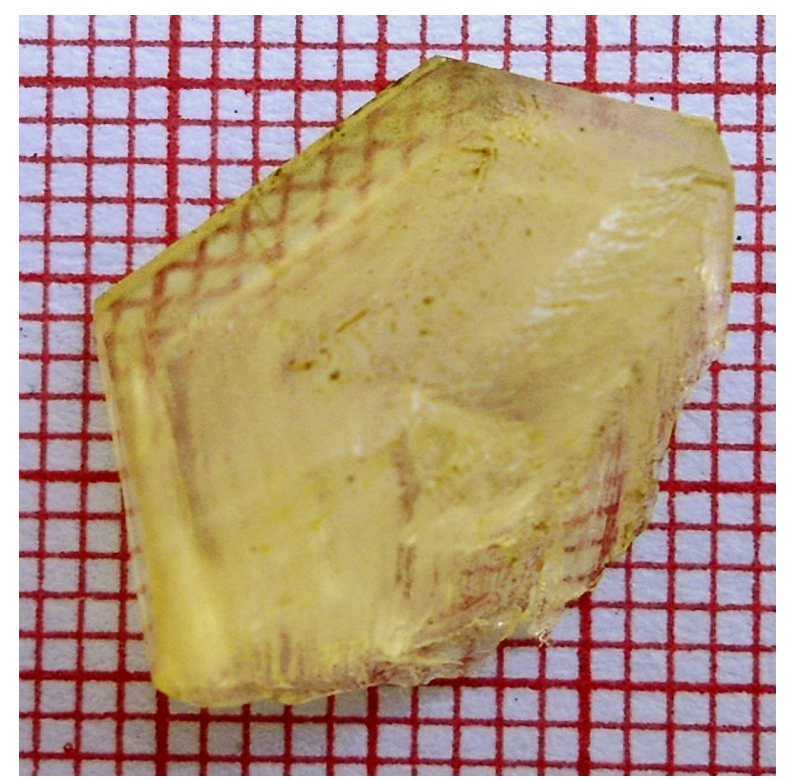

Fig. 1. Photograph of as-grown TPMNA crystal.

\section{Results and discussion}

\subsection{Powder XRD analysis}

Powder X-ray diffraction patterns recorded for the grown crystals by a SHIMADZU XRD 6000 $\mathrm{X}$-ray diffractometer are shown in Fig. 2. Comparison of TPM [10] and 4NA (JCPDS Card
No. 38-1962) with the XRD pattern of TPMNA reveals that the grown TPMNA has a different structure and new composition. Miller indices estimated by powder Crysfire software along with $2 \theta$ values of triphenyl methane and TPMNA crystals are given in Table 1. The cell parameters of the TPMNA crystal indicate that it belongs to triclinic crystal system. The calculated cell parameters are given in Table 2.
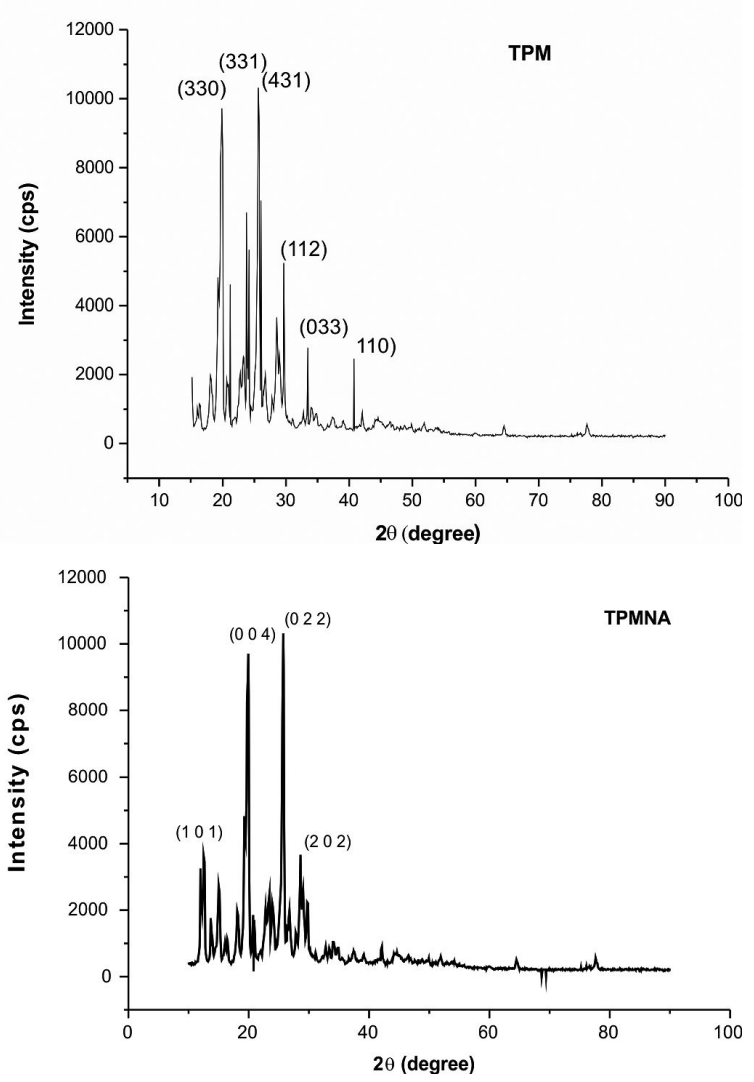

Fig. 2. Powder XRD patterns for TPM and TPMNA crystals.

\subsection{FT-IR spectral study}

FT-IR spectrum of TPMNA crystal was recorded in the range of $400 \mathrm{~cm}^{-1}$ to $4000 \mathrm{~cm}^{-1}$ using IFS BRUKER FT-IR spectrometer and it is shown in Fig. 3. The band observed at $3019 \mathrm{~cm}^{-1}$ is due to the $\mathrm{C}-\mathrm{H}$ stretching of aromatic ring. Generally, the $\mathrm{N}-\mathrm{H}$ stretching vibrations show absorption in the range of $3140 \mathrm{~cm}^{-1}$ to $3500 \mathrm{~cm}^{-1}$. A band observed at $3298 \mathrm{~cm}^{-1}$ is due to the $\mathrm{N}-\mathrm{H}$ stretching vibrations. Symmetric 
Table 1. Powder XRD data of TPM, 4NA and TPMNA crystals.

\begin{tabular}{|c|c|c|c|c|c|c|c|c|c|c|c|}
\hline \multicolumn{4}{|c|}{ TPM } & \multicolumn{4}{|c|}{$\begin{array}{c}\text { 4NA (JCPDS Card No. } \\
\text { 38-1962) } \\
\end{array}$} & \multicolumn{4}{|c|}{ TPMNA } \\
\hline $2 \theta$ & $\mathrm{h}$ & $\mathrm{k}$ & 1 & $2 \theta$ & $\mathrm{h}$ & $\mathrm{k}$ & 1 & $2 \theta$ & $\mathrm{h}$ & $\mathrm{k}$ & 1 \\
\hline 20.7274 & 0 & 3 & 3 & 12.439 & -1 & 0 & 1 & 11.994 & 0 & 1 & 0 \\
\hline 21.7645 & 0 & 1 & 6 & 12.708 & 1 & 0 & 1 & 12.472 & 1 & 0 & 1 \\
\hline 22.6060 & 1 & 3 & 2 & 14.367 & 2 & 0 & 0 & 13.795 & -1 & 0 & 1 \\
\hline 22.7829 & 0 & 3 & 4 & 16.326 & -1 & 1 & 0 & 15.879 & 1 & 1 & 0 \\
\hline 23.5611 & 2 & 0 & 0 & 17.956 & 0 & 1 & 1 & 16.368 & 1 & 1 & 2 \\
\hline 23.9240 & 2 & 0 & 1 & 19.275 & -1 & 1 & 1 & 18.159 & -1 & 1 & 0 \\
\hline 24.2580 & 1 & 2 & 5 & 19.433 & 1 & 1 & 1 & 19.193 & 1 & -1 & 1 \\
\hline 24.6408 & 2 & 0 & 2 & 20.595 & -2 & 1 & 0 & 20.791 & 0 & 0 & 4 \\
\hline 25.0278 & 2 & 1 & 1 & 20.683 & 0 & 0 & 2 & 22.599 & 1 & 0 & 4 \\
\hline 25.3256 & 2 & 1 & 2 & 22.914 & -2 & 1 & 1 & 23.264 & -1 & 1 & 3 \\
\hline 25.6886 & 1 & 3 & 4 & 23.254 & 2 & 1 & 1 & 23.974 & 0 & 2 & 2 \\
\hline 26.2436 & 0 & 4 & 3 & 23.771 & -3 & 0 & 1 & 25.095 & 2 & 0 & 2 \\
\hline 26.5267 & 2 & 2 & 0 & 24.238 & 3 & 0 & 1 & 25.683 & 2 & 1 & 0 \\
\hline 26.8261 & 2 & 2 & 1 & 24.971 & 2 & 0 & 2 & 26.399 & 0 & -1 & 4 \\
\hline 27.1194 & 1 & 4 & 1 & 25.450 & 0 & 1 & 2 & 26.707 & 2 & 1 & 3 \\
\hline 27.7213 & 0 & 3 & 6 & 25.269 & 2 & 0 & 2 & 27.795 & -2 & 0 & 2 \\
\hline 28.4909 & 2 & 1 & 4 & 26.228 & 3 & 1 & 0 & 28.492 & -1 & 2 & 0 \\
\hline 28.7431 & 2 & 2 & 3 & 26.338 & -1 & 1 & 2 & 29.026 & 2 & 1 & 4 \\
\hline 28.8287 & 1 & 4 & 3 & 26.619 & 1 & 1 & 2 & 29.684 & 1 & -2 & 1 \\
\hline 30.0384 & 2 & 3 & 1 & 28.054 & -3 & 1 & 1 & - & - & - & - \\
\hline 30.8678 & 2 & 3 & 2 & 28.456 & 3 & 1 & 1 & - & - & - & - \\
\hline 32.6640 & 1 & 5 & 0 & 28.975 & 4 & 0 & 0 & - & - & - & - \\
\hline 33.3272 & 1 & 5 & 2 & 29.091 & 2 & 1 & 2 & - & - & - & - \\
\hline 33.8665 & 0 & 2 & 9 & 29.604 & 0 & 2 & 0 & - & - & - & - \\
\hline 34.1249 & 2 & 2 & 6 & 30.494 & -1 & 2 & 0 & - & - & - & - \\
\hline
\end{tabular}

and asymmetric stretching of $\mathrm{NO}_{2}$ group give the peaks at $1490 \mathrm{~cm}^{-1}$ and $1511 \mathrm{~cm}^{-1}$ [11]. The peak at $1723 \mathrm{~cm}^{-1}$ confirms the existence of functional group $\mathrm{C}-\mathrm{C}=\mathrm{C}$ symmetric stretching $[12,13]$. The group assignments confirm the chemical structure of the grown crystal.

\subsection{UV-Vis-NIR spectral study}

UV-Vis absorption was performed to study optical properties of the grown TPMNA crystal. The UV-Vis-NIR spectrum of TPMNA was documented in the wavelength range of $200 \mathrm{~nm}$ to $1100 \mathrm{~nm}$ using Agilent technology Cary $60 \mathrm{UV}-\mathrm{Vis}$ nano-spectrometer and it is shown in Fig. 4. Optical absorption study shows that the cut-off wavelength of TPMNA is $294 \mathrm{~nm}[14,15]$.
The band gap energy of TPMNA was calculated from the equation:

$$
E_{g}=\frac{\hbar c}{\lambda}
$$

where $\mathrm{h}$ is the Planck constant $=6.626 \times 10^{-34} \mathrm{~J} / \mathrm{s}$, $\mathrm{c}$ is the speed of light $=3.0 \times 10^{8} \mathrm{~m} / \mathrm{s}, \lambda$ is cut-off wavelength $=294.007 \times 10^{-9} \mathrm{~m}$. Band gap energy value of the TPMNA crystal was found as $6.625 \mathrm{eV}$.

\subsection{Vickers microhardness studies}

Vickers microhardness technique is used to study the elastic and plastic nature and to determine the mechanical strength of a material. Vickers microhardness tests were carried out by 
Table 2. Comparison of unit cell parameters of TPM, 4NA and TPMNA crystals.

\begin{tabular}{lccc}
\hline Unit cell parameters & TPMNA & Pure TPM & $\begin{array}{c}\text { 4NA (JCPDS } \\
\text { Card No. } \\
38-1962)\end{array}$ \\
\hline \hline $\mathrm{a}$ & $7.499714 \AA$ & $7.52 \AA$ & $12.318 \AA$ \\
$\mathrm{b}$ & $7.688077 \AA$ & $14.80 \AA$ & $6.041 \AA$ \\
$\mathrm{c}$ & $17.819270 \AA$ & $25.58 \AA$ & $8.5828 \AA$ \\
$\alpha$ & $95.36^{\circ}$ & $90.00^{\circ}$ & - \\
$\beta$ & $79.93^{\circ}$ & $90.00^{\circ}$ & $91.41^{\circ}$ \\
$\gamma$ & 80.19 & $90.00^{\circ}$ & - \\
volume & $970.17\left[\AA^{3}\right]$ & $2847\left[\AA^{3}\right]$ & $637.71\left[\AA^{3}\right]$ \\
Crystal system & triclinic & orthorhombic & monoclinic \\
\hline
\end{tabular}

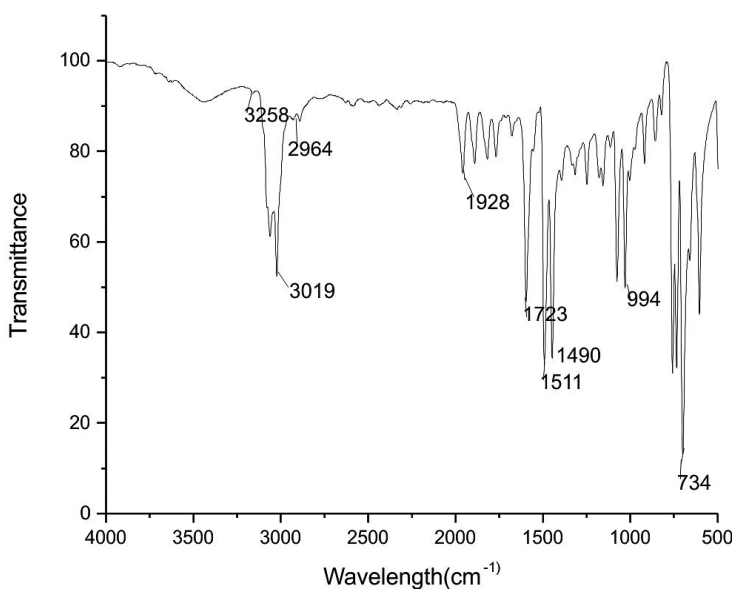

Fig. 3. FT-IR spectrum for TPMNA.

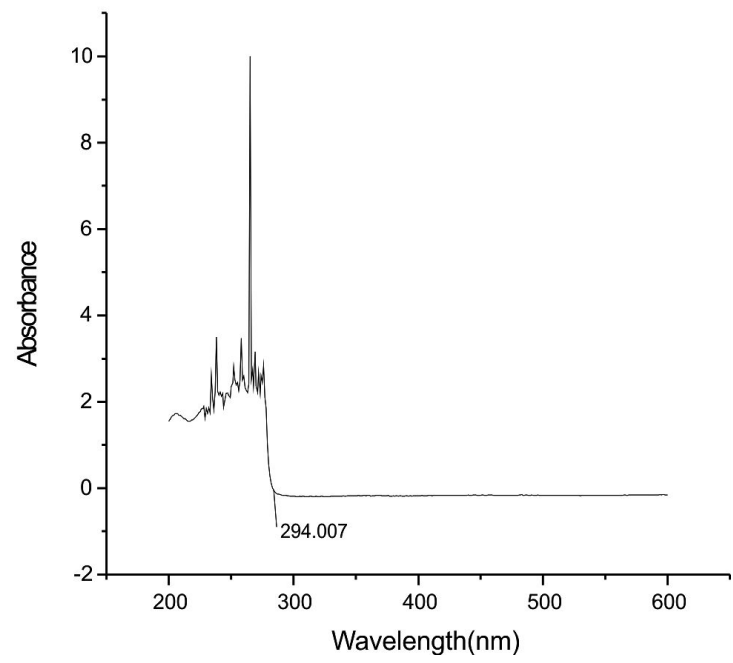

Fig. 4. UV-Vis spectrum of TPMNA. placing the crystal over a platform and applying a load of different magnitude to the samples. Using the relation given below, the hardness value has been calculated:

$$
H=\frac{1.8544 \times P}{d^{2}} \frac{\mathrm{Kg}}{\mathrm{mm}^{2}}
$$

where $\mathrm{P}$ is the magnitude of a load applied to the sample and $d$ is the diagonal length of the impression created by the indenter.

The plots of hardness number $\mathrm{H}_{\mathrm{v}}$ versus load $\mathrm{P}$ and $\log \mathrm{P}$ versus logd are shown in Fig. 5.

Up to $50 \mathrm{~g}$, the hardness value increases with an increase of load; after that cracks have been developed due to the internal stress created by the indenter. Generally, work hardening coefficient value of the harder materials lies between 1 and 1.6, and is greater than 1.6 for softer materials [16]. The hardening coefficient value of TPMNA is around 2.7692 and it reveals that the grown crystal has a soft nature $[17,18]$.

\subsection{Dielectric measurements}

Dielectric measurements were carried out for the grown sample in function of frequency and temperature. The dielectric constant has been calculated by using the following expression:

$$
\varepsilon=\frac{C d}{\varepsilon_{0} A}
$$

where $\mathrm{C}$ is the capacitance value, $\mathrm{A}$ and $\mathrm{d}$ are the area and thickness of the sample, respectively. 


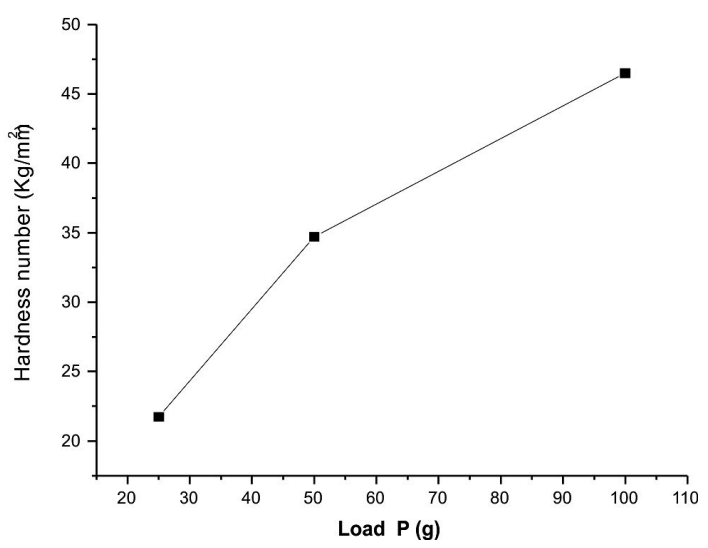

(a)

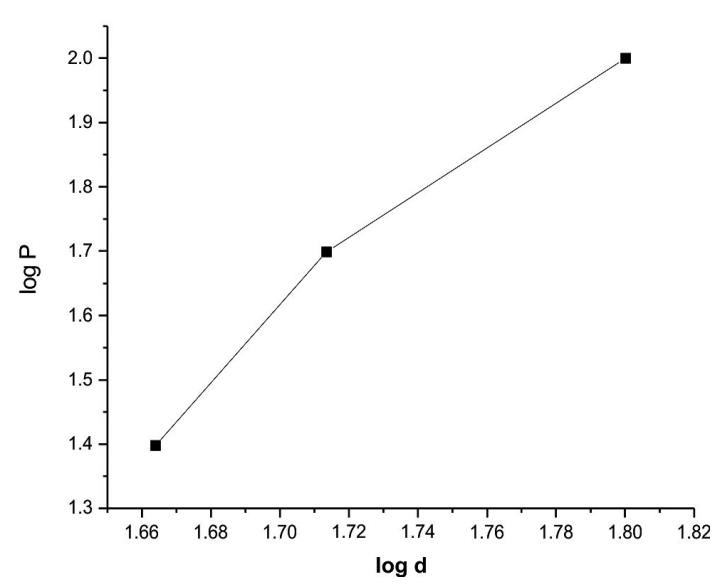

(b)

Fig. 5. Vickers hardness number $\mathrm{H}_{\mathrm{v}}$ versus load (a), $\log \mathrm{P}$ versus $\log \mathrm{d}(\mathrm{b})$.

Generally, in dielectric materials, application of electric field causes polarization. Electronic, ionic, orientation and space charge polarization can be observed at the time of applying the electric field to a material. From this polarization mechanism the relative permittivity and dielectric loss of the material were calculated. The dielectric loss $\epsilon_{\mathrm{r}}^{\prime \prime}$ factor was calculated by using the relation:

$$
\varepsilon_{y}^{\prime \prime}=\varepsilon_{y}^{\prime} \tan \delta
$$

The plots of dielectric constant and dielectric loss versus $\log$ frequency for TPMNA are shown in Fig. 6a and Fig. 6b.

It is observed from the Fig. 6a and Fig. 6b that the values of dielectric constant and loss

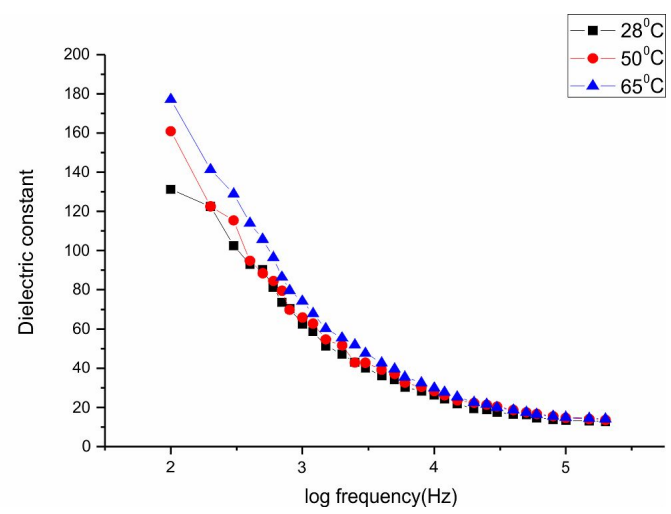

(a)

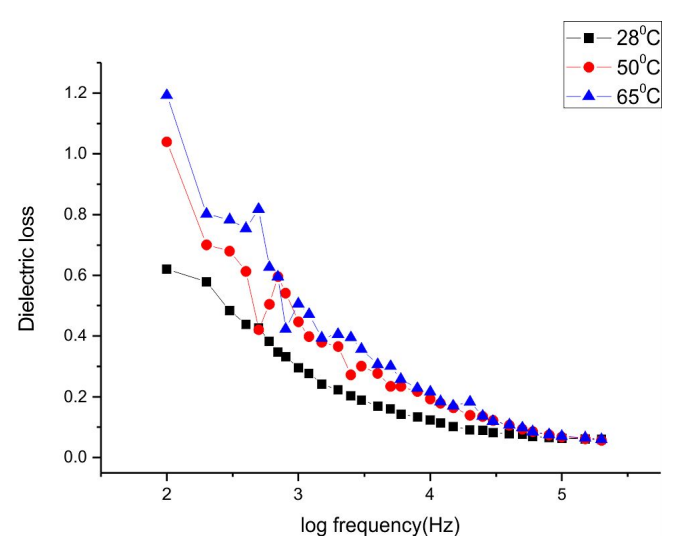

(b)

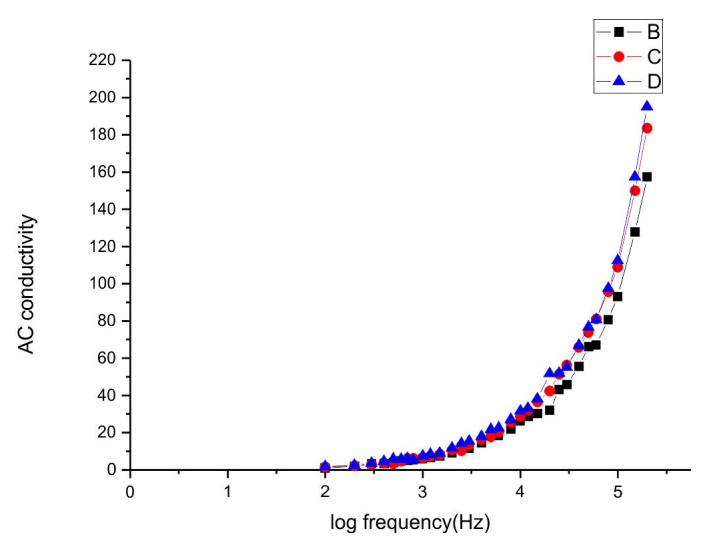

(c)

Fig. 6. Dielectric constant (a), dielectric loss (b) AC conductivity (c) vs. log frequency for TPMNA.

decrease gradually when the frequency increases. This is a behavior of a typical dielectric material. The high value of dielectric constant at lower frequencies region may be attributed to the occurrence of space charge polarization $[19,20]$. 
These curves suggest that the grown crystal is suitable for the frequency conversion process. The AC conductivity has also been calculated as a function of applied frequency using the following relation:

$$
\sigma_{a c}=\varepsilon_{r} \varepsilon_{0} \omega \tan \delta
$$

where $\epsilon_{0}$ and $\omega$ are permittivity of free space and angular frequency $(\omega=2 \pi v)$ of the applied field, respectively. The dependence of AC conductivity on $\log \mathrm{f}$ is shown in Fig. 6c. It is seen that the AC conductivity increases with an increase in the applied frequency [21].

\subsection{NLO studies}

The second harmonic generation (SHG) properties of a grown crystal of TPMNA have been examined using the input energy beam of Nd:YAG laser $(\lambda=1068 \mathrm{~nm}), 1.2 \mathrm{~mJ} /$ pulse with a pulse duration of $10 \mathrm{~ns}$ and frequency repetition of $10 \mathrm{~Hz}$ to a microsize crystalline powder sample. Emission of green beam confirmed the SHG properties of the grown crystal. The power output of the microcrystalline sample is comparable to the standard KDP The standard KDP crystal gives a SHG signal of $24 \mathrm{mV}$ for the same input energy. TPMNA crystal gives a SHG signal of $30 \mathrm{mV}$. The SHG efficiency of TPMNA is 1.25 times higher than that of KDP crystal [22].

\section{Conclusions}

Good quality single crystals of triphenyl methane 4-nitroaniline (TPMNA) have been grown at room temperature. Structural characterization of the grown crystals confirmed that the obtained crystal has different crystal system compared to the parent materials. The presence of functional groups and their vibrational character has been studied. It has been confirmed by the optical spectra analysis that the crystal has wider transparency in the visible and NIR region; also the band gap energy value has been found as $6.625 \mathrm{eV}$. The microhardness analysis revealed that triphenyl methane belongs to soft material category. Dielectric nature of the grown crystal was studied by varying the frequency of the electric field and the temperature. SHG emission from the sample was confirmed by observing the green radiation in Kurtz and Perry technique.

\section{References}

[1] Chemla D.S., Zyss J., Nonlinear optical properties of organic molecules and crystals, Academic Press, New York, 1987.

[2] PRASAD P.N., Williams D.J., Introduction to nonlinear optical effects in organic molecules and polymers, Wiley, New York, 1991.

[3] Hari Singh Nalwa, Seizo Miyata, Nonlinear optics of organic molecules and polymers, CRC Press, Boca Raton, 1997.

[4] Mohan J., Organic Spectroscopy, principles and applications, Alpha Science International Ltd, Harrow, 2004.

[5] KeMP W., Organic spectroscopy, Palgrave Macmillan New York, 1993.

[6] KALSI P., Spectroscopy of organic compounds, Willey Eastern, New Delhi, 1985.

[7] DANi V.R., Organic spectroscopy, Tata Mc Graw Hill, New Delhi, 1995.

[8] Barzoukas M., Josse D., Fremaux P., Zyss J., Nicoud F., Morley J.O., J Opt. Soc. Am. B., 4 (1987), 977.

[9] http: //www. chemicalbook.com/ ChemicalProductProperty_EN_CB5689132.htm.

[10] Mejeba M.X., Devarajan P.A., In. J. Eng. Res. Tech., 4 (2015), 952.

[11] Prasad G.L., Krishnakumar V., Nagalakshmi R., Spectrochim. Acta A, 110 (2013), 377.

[12] Boopathi K., Ramasamy P., Optik, 126 (2015), 2125.

[13] Marder S.R., KipPelen B., Jen A.K.Y., Peyghambarian N., Nature, 388 (1997), 845

[14] Katz H.E., Singer K.D., Sohn J.E., Dirk C.W., King L.A., Gorden H.M., J. Am. Chem. Soc., 109 (1987), 6561.

[15] Breslow R., Chu W., J. Am. Chem. Soc., 92 (1969), 2165.

[16] Jagadeesh M.R., Suresh Kumar H.M., Ananda Kumar R., Mater. Sci.-Poland, 33 (2015) 529.

[17] Renfrow Jr. W.B., Hauser C.R., Org. Synth. Coll., 2 (1943), 607.

[18] NorRIS J.F., Org. Synth. Coll., 1 (1941), 548.

[19] Sangwal K., Mater. Chem. Phys., 63 (2000), 145.

[20] Ikeda H., Sakai T., Kawasaki K., Chem. Phys. Lett, 179 (1991), 551.

[21] Dmitriev V.G., Gurzadyan G.G., Nikogosyan D.N., Nonlinear Optical Crystals, Springer, Berlin 1991.

[22] Kurtz S.K., Perry T.T., J. Appl. Phys., 39 (1968), 39.

Received 2017-04-26 Accepted 2017-08-17 